\title{
Climate change management: a resilience strategy for flood risk using Blockchain tools
}

\author{
Emanuele Vannucci ${ }^{1}\left[\right.$ - Andrea Jonathan Pagano $^{2} \cdot$ Francesco Romagnoli $^{2}$
}

Received: 8 May 2020 / Accepted: 29 November 2020 / Published online: 1 March 2021

(C) The Author(s) 2021

\begin{abstract}
This work aims to offer a contribution in the analysis and management, from an economic and financial point of view, of the flood risk, and extended to the hydrogeological risk, from the perspective of a public administration. As main responsible actor for containing the phenomenon through the maintenance of the territory, public administration is responsible for the cost of restoring of the services that have been damaged by this type of phenomenon. The assets of which the public administration must ensure the restoration are all public infrastructures (i.e. transportation, energy and water supply system, communication) together with the damage suffered by private property, if these affect services to be guaranteed to the population. In this work, the authors propose possible strategies that a public administration can put in place to deal with flood risk. Three main strategies are analysed: an absolute passivity that provides for the payment of damages as they occur (i.e. business-as-usual scenario), a classic insurance scheme, a resilient and innovative insurance scheme. The economicfinancial profiles of these strategies proposed in this work put an emphasis on how the assumption of a time horizon can change the convenience of one strategy compared to the others. This study highlights the key role of the quantification of flood risk mitigation measure from an engineering perspective, and their potential issues to pursue these objectives in connection to the regulatory framework of the public administrations. This synergy is supported by the potential use of Blockchain-based tools. Within the paper is highlighted the key role that such platform IT data management platform could have within risk analysis and management schemes, both as a data collection tool and as certification of the various steps necessary to complete the process.
\end{abstract}

Keywords Climate change $\cdot$ Flood risk $\cdot$ Insurance $\cdot$ Resilience $\cdot$ Blockchain

\footnotetext{
Emanuele Vannucci emanuele.vannucci@unipi.it

1 Department of Economics and Management, University of Pisa, Pisa, Italy

2 Institute of Energy Systems and Environment, Riga Technical University, Riga, Latvia
} 


\section{Introduction}

The phenomenon of climate change is intensifying the manifestations of extraordinary climatic events, and in recent decades, worldwide, the damage has raised to tens of billions of dollars. The climatic phenomena that cause damage are different depending on the geographical areas. These have already been largely addressed within the insurance market (Munich 2013), both as traditional products and with mechanism involving the financial markets, i.e. cat-bonds. Such mechanism encountered an important increase in the market since their appearance a few decades ago.

In addition to the insurance market, focused on the transfers the risk and part of the payments to other subjects, in several risk reduction strategies it is mostly the public administration that ultimately has to face the management of these risks and the consequent damages unlocking a significant and growing economic challenge on how to face with up-front investment for adaptation or mitigation risk reduction solutions (UNISDR 2015).

A particular type of risk related to extreme climatic phenomena is flooding both as riverine or coastal, which is aggravated with the increase in the number of extreme climate event such heavy rains or storm surges. The impact on the local assets depends on the local conditions including morphology of the territory and its underlying hydrogeological risk, the overall vulnerability of the population and the infrastructures exposed to the hazard. Specifically, hydrogeological risk is a type of risk that, in addition to private assets, creates damage to many public infrastructures (e.g. transportation network, energy and water distribution systems, communication network) that the public administration it is forced to restore, often at very high costs.

The theme we want to present in this paper proposes a financial scheme of a flood risk management process from the point of view of a public administration, which can choose between different risk reduction strategies.

The first is the completely passive payment of the damages as they occur (i.e. business-as-usual scenario). The second is a traditional insurance scheme. The third entails an attitude of urban resilience, which consists in evaluating the possible convenience of up-front investments for risk mitigation through an appropriate hazard mitigation or adaptation project specifically addressed to have a more resilient urban infrastructures and assets at risk.

This third strategy follows the idea of the financial structure as proposed by the socalled resilience bonds, which are having a first development in recent years starting from the first issue in 2019 of a five-year climate resilience bond by the European Bank for Reconstruction and Development (EBRD).

In this paper, a comparative quantitative assessment model of the three proposed strategies will be presented, based on a stochastic process that can describe the expectations of the level of damage that will be achieved over future time horizons. A similar quantitative-based approach has been presented in recent previous papers by the same authors, Pagano et al. (2019a,b), and by Reguero et al. (2020) for financing coastal resilience.

The problem analysis will be completed by describing the other disciplines involved in this risk management scheme, i.e. the role of engineering competence will be explained, in terms of risk assessment and costs-benefits (in terms of mitigation respect 
to the original level of risk) of the infrastructures that could be used for this purpose (embankments, dams, expansion tanks, ...) and also what is the regulatory framework (particularly for the European context) within the public administrations can act to pursue these objectives.

Finally, this paper wants to create the ground on the use of an innovative IT technology platform for the insurance sector (i.e. Blockchain). Blockchain-based tools provide the interface for real-time climate data collection and registered damages. The platform provides the automatic certification of the acquired information within each steps of the process, both in terms of the regulatory and financial framework, and in terms of the implementation of risk mitigation infrastructures.

This paper has the following structure.

The first paragraph describes the regulatory context in which the public administration can act to deal with flood risk and catastrophic risks in general. The second paragraph is dedicated to comparing the convenience of the various strategies that the public administration can implement according to a quantitative actuarial scheme, even with a numerical example in order to offer a sensitivity analysis for some key parameters, with the crucial role of engineering expertise for their assessment. The third paragraph will highlight the possible role of Blockchain in the various steps of the flood risk management scheme presented in this work. Finally, some conclusions and ideas for further research developments will be pointed out.

\section{Public administrations and the regulatory framework for flood risk assessment and management}

Since the early 1970s, extreme events associated with natural disaster have been growing both in frequency and intensity. Specifically, during the last 15 has been recorded an increase of 2\% per year as reported in Serre and Heinzlef (2018).

The same increased trend was also reflected on flood disasters registered from 2007. What happens in the year 2013 in the Central Europe was particularly impactful: 16.5 billion in economic losses (large-scale damage across Germany, the Czech Republic, Hungary and Poland) for 4.1 billion in insurance paid claims. The year 2013 has the negative record of the increase in flood damages of approximately $50 \%$ respect to the period 2003-2012 and to show for the first time three consecutive losses exceeding 100 billion in a 10 years period time (Swiss 2014).

These figures represent an evidence how the increase in the population in urban areas and the consequential increase in their complexities of both social and technological dimensions define a bottleneck within flood risk management in public administration.

In fact, the rapid growth of human concentration and urbanized areas has increased the exposure to the existing flood recurrence time making more difficult the realization of proper mitigation measures such as the availability of the land to be settled as potential flood risk zone, or protection and improve safety of river banks.

Among different assets that increased their risk to flood due to an increased exposure, Critical Infrastructures (CI) need a specific emphasis. Critical infrastructures represent body of systems, networks and assets that are essential for the functioning of a society, public's health and/or safety and economy of a nation. CI are thus engineer- 
ing and technological networks, such as energy and water supply systems, transport services, banking and finance, and ICT (information and communication technology) systems. All these systems are important (and thus critical) to maintain essential functions of society, and their failures can heavily seriously affect the population, economy and national security [as stated in Galland (2010) and in Serre (2018)].

This is the reason that addressed the attention of policy-makers, economist, urban planners, engineers, insurance companies and scientist to find innovative risk management frameworks to more sustainable and more resilient cope with climate changes effect and natural hazards (Quenault 2014).

There are initiatives at various levels worldwide with the aim of creating coordination and guidelines for public administrations, to design resilient schemes in the face of the risks of climate change, also with regard to the economic-financial aspects of risk assessment and financing of resilient actions.

Keenan (2019) describes the reference legislation and the opportunities granted by it, to adopt resilient strategies against the risks associated with climate phenomena in that area as exposed as California is.

Also in the European context, significant steps towards a coordination of local administrations have been observed, mostly in the assessment of risks deriving from the climate change phenomenon.

In particular, in 2008, the European Commission launches the first, and to date the most ambitious, initiative targeting local and subnational authorities to lead climate and energy action. As part of the Covenant of Mayors, cities and towns take action towards sustainable energy, including alleviation of energy poverty, climate change mitigation and adaptation to secure a better future for their citizens.

Covenant of Mayors for Climate and Energy (2008) is a voluntary-based initiative focused on the proactive role of local authorities for making territories (more) resilient to the impacts of climate change. That work produced Sustainable Energy and Climate Action Plan (SECAP) in 2015 (Bertoldi 2018).

One key point of the SECAP is Risk and Vulnerability Assessment (RVA), which is an analysis of the relevant risks and vulnerabilities, by analysing climate hazards and assessing vulnerability (of urban sectors): (1) Municipal buildings, equipment/facilities, (2) Tertiary (non-municipal) buildings, equipment/facilities, (3) Residential buildings, (4) Transport.

The assessment could address for instance risks related to floods, extreme low temperatures and heat waves, droughts and water scarcity, storms and other extreme weather events, increased number or intensity of forest fires, sea level rise and coastal erosion.

In a SECAP, a Baseline Emission Inventory and a Risk and Vulnerability Assessment are mandatory and identify a set of actions to be undertaken: one of these actions could be the introduction of resilience bonds to finance risk mitigation strategy by a more resilient infrastructural system.

A joint SECAP can be developed by a group of adjoining local authorities. This can even increase the effectiveness of the risk reduction plan by the definition of a regional common long-term vision. 


\section{A quantitative comparison among different risk management strategies}

This paragraph will describe the financial schemes generated by three different strategies for dealing with flood risk by the public administration:

- the passive strategy provides for the payment of damages as they occur,

- the standard insurance strategy provides for the quantification of a premium with which to transfer the burden of compensation (in part or totally) to the insurance market,

- the innovative insurance resilient strategy, involves combining the standard insurance scheme with the financing of mitigating infrastructures, which will reduce risk exposure once completed.

It will therefore be a question of comparing the effectiveness of the different strategies, based on the time horizon that will be set, taking into account that the advantage of the resilient strategy will materialize after the mitigating infrastructures, which involved higher costs in the initial phase, will be completed. We consider a similar approach to one proposed in Reguero et al. (2020), but we assume a stochastic framework for the damages.

Flood risk reduction by an enhanced urban resilience means moving towards the improvement of mitigative infrastructures (e.g. hydraulic defence works, retaining dams, expansion tanks, ...). Within this process is required the risk assessment through engineering modelling including the calculation of potential losses before and after the realization of a mitigation project and the overall costs of and time required to build the resilient infrastructure. This aspect is not trivial since each engineering solution and its risk reduction assessment is site specific and not always straightforward.

Within the proposed model, the authors refer to the original source of the flood risk in connection to, that is to rain levels and/or to those of river beds in the area exposed to the risk.

It would be a question of finding the form of dependence, through statistical inference issues, of the damages recorded starting from the data of this primary source of risk, which can be a not easy task since the quality of the databases linking the damages to corresponding climate phenomena are not so good, almost for many public administrations archives. Also other study Reguero et al. (2020), regarding the economic loss distribution for coastal damages, no reference is made to any database of actually recorded losses.

Therefore, it was decided to skip this aspect of the investigation, which would have removed us from the focus treated in the present work.

We will directly acquire the knowledge of a historical series of the damages recorded, with the temporal scan (year, season, month, ...) which will be at the basis of the quantitative model: for simplicity and to avoid seasonality phenomena relating to the climatic events in question, we will consider an annual basis, which is a usual assumption in many actuarial models. In what follows, therefore, all the quantities will be considered defined on an annual basis. 


\subsection{The model of risk exposure}

Let $X(h)$, i.i.d for $h=1,2, \ldots$, the yearly random payment for flood damages in year $h$, with distribution function $f(X)$, that is $f(X)=f(X(h)) \forall h$, which can be estimated by the analysis of historical series of yearly damages, with moments $E\left[X^{r}\right]$, for $r=1,2, \ldots$.

Let assume an insurance premium function of $f(X)$, that is $P=g(f(X)), g: \Re \rightarrow$ $\Re$, for which a standard assumption due to a risk aversion principle, is $P>E[X]$. We assume a full coverage of the damages by the insurance contract.

Assume that with a cost $W$ and a completion time $n$, a mitigative infrastructure provides that the r.v. which describes the yearly damage for following years is $X_{R}$ such that, $E\left[X_{R}\right]<E[X]$ and $\sigma\left[X_{R}\right]<\sigma[X]$, from which for the insurance premium with the same function $g$, it holds $g\left(f\left(X_{R}\right)\right)=P_{R}<P$.

The assessment of risk reduction by engineering expertise could be a hard task, since it cannot be evaluated using historical series of damages (the mitigative infrastructure did not exist before).

\subsection{The comparison of strategies expected costs over a time horizon}

In this paragraph, we will proceed to the comparison of the expected costs of the three different strategies, fixed the time horizon. Since the comparison must be made in terms of current values, a generic annual discounting factor $v$ corresponding to the rate of $i$ must be fixed, that is $v=(1+i)^{-1}$.

For the passive strategy (indicated with the subscript $\mathrm{P}$ in the following symbols), the random present value of the total payment by the public administration, fixed a generic time horizon of $m$ years, $C_{P}(0, m)$, is

$$
C_{P}(0, m)=\sum_{h=1}^{m} X_{h} v^{h}
$$

from which its expected value is a deferred annuity of instalment $E[X]$, that is

$$
E\left[C_{P}(0, m)\right]=\frac{1-v^{m}}{i} E[X]
$$

For the standard insurance strategy (indicated with subscript I in the following symbols), the current value of the total expenditure for the public administration, which is deterministic in this case, is a deferred annuity of instalment $P$, that is

$$
C_{I}(0, m)=\frac{1-v^{m}}{i} P
$$

For risk aversion principle, from which it follows $P>E[X]$, we have

$$
E\left[C_{P}(0, m)\right]<C_{I}(0, m)
$$


but the passive strategy could incur in annual compensation so high as to endanger the financial solidity of the public administration, which instead, with the insurance strategy, can plan a constant yearly payment equal to $P$. The probability of very high compensation increases as the volatility of $X$ increases, that can be deduced from the historical series, with which its distribution $f(X)$ is estimated.

The resilient strategy (indicated with subscript $\mathrm{R}$ in the following symbols) provides that for $n$ years it will be necessary to pay the insurance coverage $P$ and to finance the mitigating infrastructures for which the cost $W$ was assumed, while after completion time the annual insurance cost decreases to the level $P_{R}$.

Therefore, let $Q$ be the annual instalment assuming that it has to be paid for the entire duration of the construction of the mitigative infrastructure (but different durations could also be considered), that is $n$ years, to finance the cost of the mitigating infrastructure, which satisfies the equation

$$
W=\frac{1-v^{n}}{i} Q
$$

from which we have the total expenditure, also in this case deterministic, incurred by the public administration for the first $n$ years

$$
C_{R}(0, m)=\frac{1-v^{n}}{i}(P+Q)
$$

and the following chain of inequalities

$$
E\left[C_{P}(0, n)\right]<C_{I}(0, n)<C_{R}(0, n)
$$

that is, in terms of expected values, in the first $n$ years the passive strategy (albeit with a random result while the other are deterministic) is more convenient than the standard insurance strategy, which in turn is cheaper than the resilient one.

It is therefore a question of studying the break-even point problem in terms of time horizon, starting from which the resilient strategy becomes more convenient than the others, taking into account that for a generic value $m>n$ the present (deterministic) value of the expenditure overall for this strategy is

$$
C_{R}(0, m)=\frac{1-v^{n}}{i}(P+Q)+v^{n} \frac{1-v^{m-n}}{i} P_{R}
$$

So the break-even point respect to the standard insurance strategy will be $m_{I}^{*}$ that is the minimum value of the time horizon $m(>n)$ such that

$$
m_{I}^{*}=\min _{m=n+1, n+2, \ldots} C_{R}(0, m)<C_{I}(0, m)
$$


while one respect to the passive strategy will be $m_{P}^{*}$ that is the minimum value of the time horizon $m(<n)$ such that

$$
m_{P}^{*}=\min _{m=n+1, n+2, \ldots} C_{R}(0, m)<E\left[C_{P}(0, m)\right]
$$

The evaluation of the cost $W$ and completion time $n$ of the mitigating work and the quantification of the risk reduction through an engineering expertise can be a complicated objective, above all because there is no real feedback on the exposure to risk following the completion of the work, but it is necessary to proceed only with hypotheses validated in contexts with some similarity.

A further development, which rests on such ability to estimate through engineering skills, could be to evaluate a possible range of mitigating infrastructures, with costs and times given by pairs $W(j)$ and $n(j)$, in the case of the generic $\mathrm{j}$-th option, $j=$ $1,2, \ldots, J$, from which the ex-post risk exposure distribution is described by the random variable $X_{R}(j)$ and the corresponding reduced premium $P_{R}(j)$.

In this case, the problem of optimizing the choice of the mitigating work could concern the minimum $P_{R}(j)$ fixed a maximum level of infrastructure cost, or the minimum in terms of break-even point provided by the different choices, that is the minimum $m^{*}(j)$, with $J \in, 1,2, \ldots J$.

In this comparison of convenience of the different strategies, the role of Blockchain tools underlying the concepts of smart contracts, would be essential for the need of automatic contract passages, from one phase to the next, without wasting time, for example, from the completion of the mitigation infrastructure, to the certification of risk exposure reduction. Indeed, a smart contract can be defined as an automatic updating of contractual conditions upon the occurrence of certain conditions to be verified through Blockchain tools.

\subsection{Numerical example: sensitivity analysis on the convenience of the resilient strategy}

The aim of this section is to present the decision-making problems that may arise in terms of choosing the risk mitigation strategy, in case the distribution of the random damage is known. As already mentioned in the introductory part of section 3, no reference is made to any actual database of damage deriving from floods, but a classic assumption in the actuarial context of a lognormal distribution for the random damage is considered. In particular, we want to highlight what could be the key role of some parameters for the sensitivity analysis of the convenience of the resilient strategy compared to the others, on the basis of the model presented in the previous section.

For the random damage $X$, we assume a lognormal distribution characterized by the parameters $\mu$ and $\sigma$ and we model risk reduction after mitigative infrastructures completion time, assuming for the residual risk $X_{R}$, a lognormal distribution with parameters $\mu_{R}=\left(1-d_{1}\right) \mu$ and $\sigma_{R}=\left(1-d_{2}\right) \sigma$. 
The insurance premium loading is assumed a proportion $\alpha>0$, of the volatility of the random damage, so the total premium is

$$
P=E[X]+\alpha \sigma[X]
$$

and analogously for the premium after the mitigative infrastructure has been built

$$
P_{R}=E\left[X_{R}\right]+\alpha \sigma\left[X_{R}\right] \text {. }
$$

Considering a standard parameterization characterizing the original risk exposure and one after the construction of the mitigative infrastructure,

$$
m u=1, \sigma=2, d_{1}=0.1, d_{2}=0.1, \alpha=0.05,
$$

note that it holds

$$
E[X]=20.08, \sigma[X]=90.01, \quad \text { from which } P=24.58
$$

and

$$
E\left[X_{R}\right]=12.42, \sigma\left[X_{R}\right]=38.09 \text { from which } P_{R}=14.33
$$

and that relating to the mitigation work and its financing

$W=100, n=5, i=0.02$ from which $Q=21.21$ (it has to be payed for the planned $n$ years of completion time).

We proceed to a sensitivity analysis of the break-even points $m_{I}^{*}$ and $m_{P}^{*}$, according to $(2 \mathrm{a})$ and $(2 \mathrm{~b})$, that is the time horizon at which the resilient strategy begins to become advantageous compared to the others, respect to variations of the most significant parameters, that is the volatility of the original risk and those relating to the mitigative infrastructure. Disregarding the description of the volatility of the results and considering them only in terms of their expected values, the standard insurance strategy is always less convenient than the passive strategy, see (1).

It should be noted that as the volatility of the original risk increases, while the breakeven point with respect to the standard insurance strategy is constantly approaching, there is no monotonous trend with respect to the passive strategy, which depends on the effect of loading the related insurance premium to this parameter, that the cost of the passive strategy, a function of the expected value alone, does not suffer in such a

Table 1 Break-even point sensitivity respect to volatility of the original risk $\sigma$

\begin{tabular}{lcc}
\hline$\sigma$ & $m_{I}^{*}$ & $m_{P}^{*}$ \\
\hline 2 & 16 & 27 \\
2.1 & 13 & 24 \\
2.5 & 7 & 22 \\
3 & 6 & 89 \\
\hline
\end{tabular}


Table 2 Break-even point sensitivity respect to mitigative infrastructures cost $C$

Table 3 Break-even point sensitivity respect to risk reductions deriving from mitigative infrastructures measured by $d_{1}=d_{2}$

Table 4 Break-even point sensitivity respect to mitigative infrastructures completion time $n$

\begin{tabular}{lll}
\hline$W$ & $m_{I}^{*}$ & $m_{P}^{*}$ \\
\hline 100 & 16 & 27 \\
110 & 17 & 29 \\
150 & 21 & 36 \\
200 & 26 & 45 \\
\hline
\end{tabular}

\begin{tabular}{lll}
\hline$d_{1}=d_{2}$ & $m_{I}^{*}$ & $m_{P}^{*}$ \\
\hline 0.1 & 16 & 27 \\
0.11 & 15 & 25 \\
0.15 & 13 & 20 \\
0.2 & 12 & 17 \\
\hline
\end{tabular}

\begin{tabular}{lll}
\hline$n$ & $m_{I}^{*}$ & $m_{P}^{*}$ \\
\hline 5 & 16 & 27 \\
6 & 17 & 29 \\
8 & 19 & 33 \\
10 & 21 & 37 \\
\hline
\end{tabular}

significant way. Of course the higher is the volatility of the original risk, the less safe is the passive strategy, since the probability of huge claims increases, that may create serious difficulties to the general economic situation of the public administration.

The results are widely expected, that is the break-even point moves away as the cost of the mitigation work increases. It could be interesting to analyse a model such that as the cost of mitigation works increases, even their effectiveness, in terms of risk reduction, increases, which could lead to a not-monotonous trend in the break-even point. However, we may have to consider a minimum level of abatement required, in order not to make the break-even point the only decision-making element in terms of measuring the efficiency of the mitigating intervention.

As regards the sensitivity with respect to the reduction of risk deriving from the mitigative infrastructure, we assume that the reduction rates of the parameters that describe the original risk, $\mu$ and $\sigma$, have the same value, that is $d_{1}=d_{2}$, whereas the effects of the mitigation works could impact the values of these parameters in very different ways, depending on the type of intervention.

It is interesting to note the effect of shortening the break-even point with increasing effectiveness, much more pronounced for the passive strategy rather than the insurance one.

It is quite clear that, given the higher cost of the resilient strategy until the completion of the mitigation work, if this period is longer, it also entails an obvious shift in the break-even point, of roughly the same magnitude compared to the standard insurance strategy and even more pronounced compared to the passive strategy. 


\section{Blockchain and the legal environment for smart contracts}

Starting from the pioneering papers of Szabo (1997, 1998), there is no universally accepted definition of smart contract and for the purpose of our paper we can use one of the most used (r2), "an agreement whose performance is automatic, so an algorithm for computer transactions, which comply with the terms of the contract".

A more detailed definition, even thinking about the applicative scope of the paper was provided by the Italian IVASS (Italian Institute for Insurance Supervision), according to which smart contracts are contracts that are written in a specific language that can be understood, translated and executed by a computer, whose clauses can produce actions without external intervention based on information received in input and processed according to predefined rules, see Grasso (2018), and the information technology underlying this automation is just the Blockchain.

About the potential (and in same cases effective) role of Blockchain in insurance environment, for various purposes, see (Gatteschi et al. 2017, 2018; Sayegh 2019; Pagano et al. 2019a, b).

Hence, a smart contract can then be thought as a multiphase contract, in which the steps to be controlled to proceed to the next phase are set at the beginning, which seems exactly the case of the mitigating process through the resilient strategy described in this work.

The various steps of the process are: the initial data collection relating to climatic phenomena (and their consequences in terms of flood phenomena) and the damage caused by them, for which the Blockchain can act in terms of certifying that the data comes from reliable sources, the stipulation of the contract both in the insurance part and in the financing part of the mitigation work, the certification of the timetable for the construction of the mitigation work (contractual clauses may be linked to any delays with respect to the settled timetable), the change in the regime of the insurance contract once the completion of the works has been certified, without the need for a new agreement on the actual exposure to risk, once this had been fixed at the signing of the contract (perhaps to be validated ex post by engineering expertise).

Note that since these mitigation processes should be of various decades and in these lengths of time some trends in climate phenomena could be observed, then the multiphase contract can consider some refreshments in the assessment of some parameters of the model, for example, ones which describe the primary risk expressed by the distribution of the random damage, with the consequent adjustment of the premium level for insurance coverage.

\section{Comments and further lines of research}

This paper presents an innovative approach on how combines the effect of up-front risk reduction investment for public administration with a resilient insurance mechanisms.

This work presented a multidisciplinary analysis on possible flood risk coping strategies, for which the entire hydrogeological risk can be understood more generally, an increasingly pressing problem for public administrations also in relation to the 
evidence of increasingly accentuated manifestations. of extreme climatic phenomena in more recent years.

The regulatory context in which local public administrations can consider possible synergies within schemes at European level (Covenant of Mayors) and in other geographical areas was described.

In addition to the use of a classic insurance approach, an assessment scheme is described deriving from a resilient approach, that is, in addition to the economic coverage of the damages that are recorded, it provides for the financing of risk mitigation works, a structure used for the so-called resilience bonds.

This paper wants to provide a consistent approach on the application of international frameworks like the Paris Agreement, the Sendai Framework for Disaster Risk Reduction and the Agenda 2030 for Sustainable Development. Moreover is well integrated in the regulatory context of SECAP.

It was highlighted that the construction of this quantitative model must be based on engineering expertise, for the ex-ante and ex-post risk assessment and for the design of the most effective mitigation works in terms of cost-benefit ratio. Given the additional cost of the mitigation work, an indicator that seems appropriate to us to compare the resilient strategy to the others is that of the break-even point, which is very common in the context of investment evaluation.

A development of the research will consist in the analysis of the quantitative scheme considering also the element of the variability of the results, for example through the use of the Monte Carlo simulation, in order to highlight how the uncertainty of the cost of claims of the passive strategy may produce much more critical scenarios than other strategies, which provide a deterministic flow for hedging risk.

The necessary multiphase process that must be completed to implement the resilient strategy, can be guided and controlled in the various phases using Blockchain tools, both in the role of data collector and in the role of certifying the completion of the various steps of the process, according to an initially established protocol that does not have to be re-discussed by the counterparties at each step, according to the scheme of so-called smart contracts.

Compared to the theoretical model presented in this work, it will now be a question of carrying out the research through the possibility of having data relating to real contexts of this type of risk, as in Castelli et al. (2019) in which a quantitative study of the flood risk of the Arno river in the Florence area is presented. The necessary data are those relating to climatic phenomena combined with those of flood damage caused by rivers that insist on the same area. It will also be necessary to know the mitigation projects that the relevant authorities already have or plan to implement in the near future, and the risk reduction assessment they plan to obtain.

The proposed approach can be exploitable and consistently applied to several types of risk and for different types of mitigation strategy strengthening the resilience of urban infrastructures against river flooding. The proposed approach provides a solution to face against lack of financial capacity for public administration that would like to sustainable and viable manage their risks. 
Further developments of the quantitative model can also be considered as a function of the quality of the data that will be available in the feasibility study of a resilient process in a real case.

Funding Open access funding provided by Università di Pisa within the CRUI-CARE Agreement.

Open Access This article is licensed under a Creative Commons Attribution 4.0 International License, which permits use, sharing, adaptation, distribution and reproduction in any medium or format, as long as you give appropriate credit to the original author(s) and the source, provide a link to the Creative Commons licence, and indicate if changes were made. The images or other third party material in this article are included in the article's Creative Commons licence, unless indicated otherwise in a credit line to the material. If material is not included in the article's Creative Commons licence and your intended use is not permitted by statutory regulation or exceeds the permitted use, you will need to obtain permission directly from the copyright holder. To view a copy of this licence, visit http://creativecommons.org/licenses/by/4.0/.

\section{References}

Bertoldi, P. (ed.): Guidebook 'How to develop a Sustainable Energy and Climate Action Plan (SECAP)'Part 1-The SECAP Process, Step-by-Step Towards Low Carbon and Climate Resilient Cities by 2030. Publications Office of the European Union, Luxembourg (2018)

Buterin, V.: A next-generation smart contract and decentralized application platform. https://github.com/ ethereum/wiki/wiki/White-Paper (2014)

Castelli, F., Galeotti, M., Rabitti, G.: Financial instruments for mitigation of flood risks: the case of Florence. Risk Anal. 39, 462-472 (2019)

Galland, J.-P.: Critique de la notion d'infrastructure critique. (Criticism of critical infrastructure concept). Flux 81, 6-18 (2010)

Gatteschi, V., Lamberti, F., Demartini, C., Pranteda, C., Santamaria, V.: Blockchain or not blockchain, that is the question of the insurance and other sectors. IT Prof. (2017). https://doi.org/10.1109/MITP.2017. 265110355

Gatteschi, V., Lamberti, F., Demartini, C., Pranteda, C., Santamaria, V.: Blockchain and smart contracts for insurance: blockchain and smart contracts for insurance: is the technology mature enough? Future Internet 10-2, 1-16 (2018)

Grasso, A.: Smart contracts: adoption value for enterprises and general use case. Digital Enterprise Magazine. https://www.thedigitalenterprise.com/ (2018)

Munich, R.: Economic consequences of natural catastrophes: emerging and developing economies particularly affected-insurance cover is essential. Position Paper, Munich, Germany (2013)

Pagano, A.J., Romagnoli, F., Vannucci, E.: Implementation of blockchain technology in insurance contracts against natural hazards: a methodological multi-disciplinary approach. J. Environ. Clim. Technol. (2019a). https://doi.org/10.2478/rtuect-2019-0091

Pagano, A.J., Romagnoli, F., Vannucci, E.: Flood risk: financing for resilience using insurance adaptive schemes. Agrochimica special issue. Pisa University Press, pp. 305-322 (2019b)

Quenault, B.: La résurgence/convergence du triptyque catastrophe-résilience-adaptation pour (re)penser la fabrique urbaine face aux risques climatiques. Développement durable et territoires 5, 3 (2014)

Reguero, B.G., Beck, M.V., Schmid, D., StadtmÜller, D., Raepple, J., SchÜssele, S., Plifiegner, K.: Financing coastal resilience by combining nature-based risk reduction with insurance. Ecol. Econ. 169, 106487 (2020). https://doi.org/10.1016/j.ecolecon.2019.106487

Sayegh, K.: Blockchain Application in Insurance and Reinsurance. Skema Business School, France (2019)

Serre, D., Heinzlef, C.: Assessing and mapping urban resilience to floods with respect to cascading effects through critical infrastructure networks. Int. J. Disaster Risk Reduct. 30, 235-243 (2018)

Serre, D.: DS3 model testing: assessing critical infrastructure network flood resilience at the neighbourhood scale. In: Fekete, A., Fiedrich, F. (eds.) Urban Disaster Resilience and Security, pp. 207-220. Springer, Cham (2018)

Swiss, R.: Natural catastrophes and man-made disasters in 2013 (2014)

Szabo, N.: The idea of smart contracts. http://szabo.best.vwh.net/idea.html (1997) 
Szabo, N.: Read related articles on Internet economics and security, pp. 1-24. https://firstmonday.org/ojs/ index.php/fm/article/view/548/469 (1998)

UNISDR (The United Nations International Strategy for Disaster Reduction): Sendai framework for disaster risk reduction 2015-2030, 36 (2015)

Publisher's Note Springer Nature remains neutral with regard to jurisdictional claims in published maps and institutional affiliations. 